\title{
Design of dual-band microstrip filter using SIR
}

\author{
Yin-Xia Zhu, Jian Zhang, Jian Cheng and Hong-Peng Zhu \\ PLA University of Sicience and Technology, Nanjing, China \\ E-mail: Zhuyinxia516@163.com
}

\begin{abstract}
Two novel dual-band microstrip filters using $\lambda_{\mathrm{g}} / 2$ Stepped-Impedance-Resonator(SIR) resonators are proposed in this paper. The prominent feature of the SIR filter is that the spurious response can be controlled by the impedance ratio $R_{\mathrm{z}}$ and the length ratio of the resonator. Simulated results show that the prototype of the dual-band filter1 achieves insertion loss of $0.4 \mathrm{~dB}$ and $0.5 \mathrm{~dB}$, return loss of $23 \mathrm{~dB}$ and $23.7 \mathrm{~dB}$, and fractional bandwidth of $9.8 \%$ and $8.2 \%$ at $2.4 \mathrm{GHz}$ and $5.2 \mathrm{GHz}$, respectively. And the filter 2 achieves insertion loss of $1.6 \mathrm{~dB}$ and $2.8 \mathrm{~dB}$, and fractional bandwidth of $6.2 \%$ and $5.1 \%$ at $2.4 \mathrm{GHz}$ and $5.2 \mathrm{GHz}$, respectively, and the isolation between two passband is well. The proposed dual-band band pass filters are designed and fabricated. A good agreement is achieved between measured and simulated results.
\end{abstract}

Keywords: Dual-band Filter; Stepped-Impedance-Resonator; Impedance Ratio; Insertion loss; Fractional Bandwidth.

\section{Introduction}

In the last few years, along with the high development and the need of wireless communications, the dual-band portable telephones and WLAN(wireless local area network) are quite popular, and the Dual-band filters become the key components in the front of these communications systems.

The main methods to realize dual-band filters are: 1.Combination of two singleband filters, however, this approach not only consumes twice the size of a single-band filter, but also requires additional impedance-matching block.[1].2.Using resonators that consist of open or short stubs in parallel or in series to create two passbands with three transmission zeros. 3. Use spurious response of coupling resonator of band pass filter.

In this paper, based on the idea put forwarded by M.Makimoto and S.Yamashita that applying the to the SIR microwave passband filter, a Dual-band filter for WLAN (IEEE-802.11 a/b/g) without any external feeds is presented, this filter consists of two cascaded resonators with $\lambda \mathrm{g} / 2 \mathrm{SIR}$, it appears very compact and easy to fabricate. The fundamental resonance frequency related to the total length of the resonator is used to create the lower passband of the Dual-band 
filter while the first spurious resonance frequency of the resonator are used to achieve the higher passband by changing its impedance ratio $\mathrm{Rz}$ and the length ratio. The impedance ratio and physical length of SIR are varied to adjust the fundamental resonance $\left(f_{1}\right)$ and the second resonance $\left(f_{2}\right)$ over a wide frequency range. The prototype of the proposed Dual-band filter is fabricated and measured. The good agreement between electromagnetic simulation and measure results shows the validity of the theory analysis and the design process.

\section{Dual-band Filter Design}

Figure 1shows the Schematic layout of the proposed dual-band filter and the basic structure of $\lambda \mathrm{g} / 2$ SIR resonators. The $\lambda \mathrm{g} / 2$ SIR resonators consists of electrical length $2 \theta_{1}$ with the characteristic impedance $Z_{1}$ and electrical length $2 \theta_{2}$ with the characteristic impedance $\mathrm{Z}_{2}, \theta_{\mathrm{t}}$ is the total electrical length of the SIR resonator.
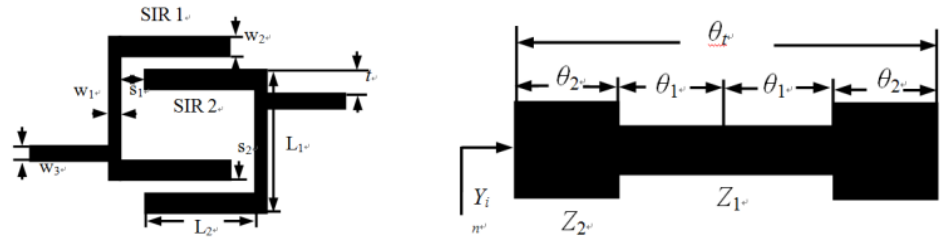

(a)

(b)

Fig. 1 (a)Schematic of the proposed dual-band filter (b)Basic structure of the $\lambda \mathrm{g} / 2$ steppedimpedance-resonator

The input admittance $Y_{\text {in }}$ of the resonator is given as .[2]:

$$
Y_{\text {in }}=-j Y_{2} \frac{2\left(R_{Z} \tan \theta_{1}+\tan \theta_{2}\right)\left(R_{Z}-\tan \theta_{1} \tan \theta_{2}\right)}{R_{Z}\left(1-\tan ^{2} \theta_{1}\right)\left(1-\tan ^{2} \theta_{2}\right)-2\left(1-R_{Z}^{2}\right) \tan \theta_{1} \tan \theta_{2}}
$$

Where $R_{\mathrm{Z}}=Z_{2} / Z_{1}$. Let $Y_{\text {in }}=0$. We can obtain the fundamental resonant frequency $f_{1}$ and the first spurious response resonant frequency $f_{2}$, from the following Eq.[3]

$$
\begin{array}{ll}
R_{Z}-\tan \theta_{1} \tan \theta_{2}=0 & \left(f=f_{1}\right) \\
\tan \theta_{2}+R_{Z} \tan \theta_{1}=0 & \left(f=f_{2}\right)
\end{array}
$$

For convenient design we choose $\theta_{1}=\theta_{2}$, define $\mathrm{u}=\theta_{2} /\left(\theta_{1}+\theta_{2}\right), \theta_{\mathrm{t}}=2\left(\theta_{1}+\theta_{2}\right)$, from the Eq. (2)and (3), we can get Eq. (4) and (5). 


$$
\begin{array}{ll}
R_{Z}-\tan \left(\frac{(1-u) \theta_{\mathrm{t}}}{2}\right) \tan \left(\frac{u \theta_{\mathrm{t}}}{2}\right)=0 \quad\left(f=f_{1}\right) \\
\tan \left(\frac{u \theta_{\mathrm{t}}}{2}\right)+R_{Z} \tan \left(\frac{(1-u) \theta_{\mathrm{t}}}{2}\right)=0 \quad\left(f=f_{2}\right)
\end{array}
$$

We note that if $R_{\mathrm{Z}}$ and $\mathrm{u}$ are confirmed, then the resonant frequency $f_{1}$ and $f_{2}$ are ensured. Figure 2(a) shows the curve of $f_{2} / f_{1}$ with the u under different $R_{\mathrm{Z}}\left(R_{\mathrm{Z}}\right.$ $=0.4,0.8,1.5$ ). Figure 2 (b) shows the curve of the total electrical length $\theta_{\mathrm{t}}$ with the u under different $R_{\mathrm{Z}}\left(R_{\mathrm{Z}}=0.4,0.8,1.5\right)$.
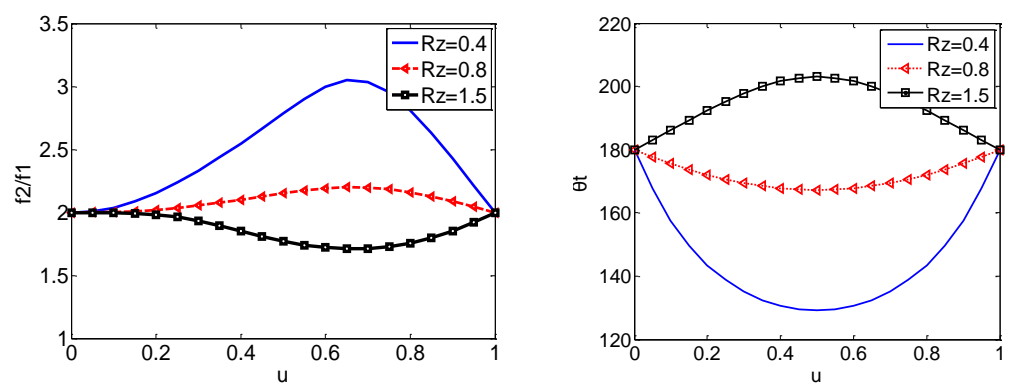

Fig. 2 (a) Curve of f2/f1 with u under different RZ (b) Curve of the $\theta t$ with u under different RZ

Fig 2(a) and (b) clearly shows that, the smaller $R_{\mathrm{Z}}$ is, the larger the maximum ratio of $f_{2} / f_{1}$ is, and the smaller the electrical length is, If $f_{2}>2 f_{1}$ is required then $R_{\mathrm{Z}} \leq 1$ should be chosen.

In this design, we choose $R_{\mathrm{Z}}=0.8, Z_{2}=50 \Omega$, then $Z_{1}=56 \Omega$. The designed two passbands of the dual-band filter are at $2.4 \mathrm{GHz}$ and $5.2 \mathrm{GHz}$, so $f_{2} / f_{1}=2.17$, from Fig 2(a) u can explicitly be determined as nearly 0.57, then from Fig 3(b), $\theta_{\mathrm{t}}$ can be determined as $168^{\circ}$.

By adjusting the structure parameters, with the help of the HFSS simulation software we can obtain a optimized dual-band filter and two dual-band filters are obtained with lower insertion loss and higher return loss between two passbands.

\section{Simulated and Measured Resulted}

The two designed filters are printed on the Rogers RO3010 substrate with thickness of $0.635 \mathrm{~mm}$ and relative permittivity of 10.2 . The filter 1 parameters are $w 1=0.45 \mathrm{~mm}, w 2=0.7 \mathrm{~mm}, w 3=0.6 \mathrm{~mm}, \mathrm{~L} 1=9 \mathrm{~mm}, \mathrm{~L} 2=6.6 \mathrm{~mm}, \mathrm{~s} 1=1 \mathrm{~mm}$, 
$\mathrm{s} 2=0.5 \mathrm{~mm}, \mathrm{t}=1.2 \mathrm{~mm}$. The total size of the filter with feeding is $27 \times 28 \times 0.635 \mathrm{~mm} 3$. The photograph of the fabricated filter is shown in Figure 3(a). The filter 2 parameters are $w 1=0.6 \mathrm{~mm}, w 2=0.9 \mathrm{~mm}, w 3=0.6 \mathrm{~mm}$, $\mathrm{L} 1=12.7 \mathrm{~mm}, \mathrm{~L} 2=4.6 \mathrm{~mm}, \mathrm{~s} 1=0.3 \mathrm{~mm}, \mathrm{~s} 2=0.25 \mathrm{~mm}, \mathrm{t}=1.2 \mathrm{~mm}$. The photograph of the fabricated filter is shown in Figure $3(\mathrm{~b})$. The total size of the filter with feeding is $28 \times 31 \times 0.635 \mathrm{~mm} 3$.

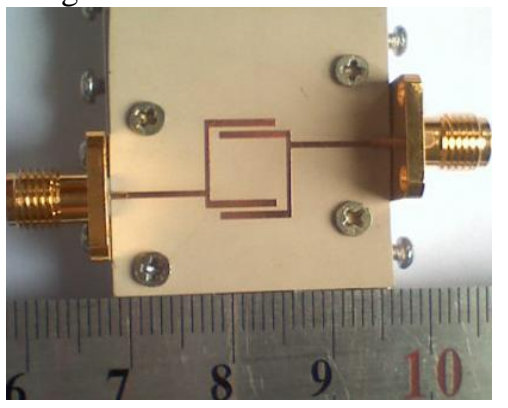

(a)

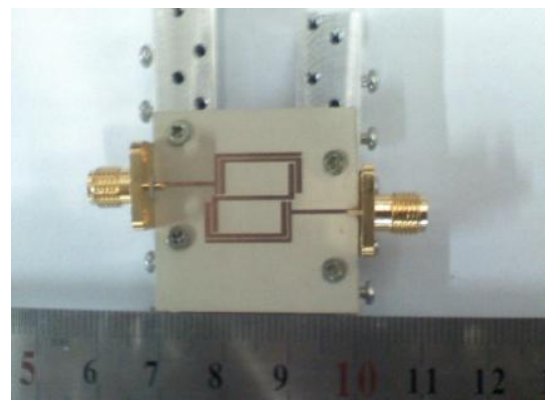

(b)

Fig. 3 Photograph of the fabricated filter .(a) filter 1. (b) filter 2

The frequency response of the proposed Dual-band filters are measured in an Agilent 8722ES network analyzer. Figure 4 shows the simulated and measured results of the two filters.

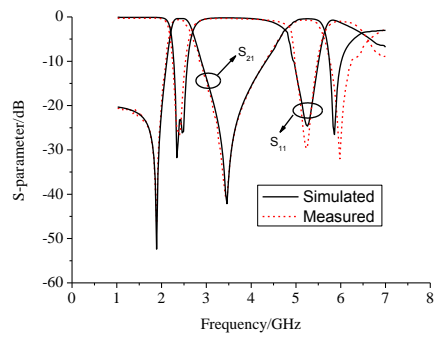

(a)

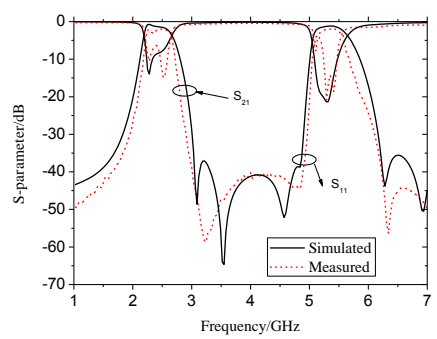

(b)

Fig. 4 Simulated and measured frequency responses of the proposed filter.(a) filter 1. (b) filter 2

For filter 1 , the simulated results show the $\mathrm{c}$ of $0.4 \mathrm{~dB}$, the return loss of $23 \mathrm{~dB}$, the Ripple coefficient of $0.1 \mathrm{~dB}$, fractional bandwidth of $9.8 \%$ at $2.4 \mathrm{GHz}$, and the insertion loss of $0.5 \mathrm{~dB}$, the return loss of $23.7 \mathrm{~dB}$, the Ripple coefficient of $0.5 \mathrm{~dB}$, fractional bandwidth of $8.2 \%$ at $5.2 \mathrm{GHz}$, besides, the transmission zero at $3.5 \mathrm{GHz}$ with insertion loss of $43 \mathrm{~dB}$ which lead to a good isolation between two 
passbands. The measurement results show the insertion loss of $0.45 \mathrm{~dB}$, the return loss of $25 \mathrm{~dB}$, the Ripple coefficient of $0.3 \mathrm{~dB}$, fractional bandwidth of $9.6 \%$ at $2.4 \mathrm{GHz}$, and the insertion loss of $0.55 \mathrm{~dB}$, the return loss of $30 \mathrm{~dB}$, the Ripple coefficient of $0.55 \mathrm{~dB}$, fractional bandwidth of $7.9 \%$ at $5.2 \mathrm{GHz}$, and the transmission zero with insertion loss of $45 \mathrm{~dB}$ is obtained at $3.5 \mathrm{GHz}$.

For filter2, in the first pass band of $2.4 \mathrm{GHz}$, the insertion loss of the simulation results and test results were $1.6 \mathrm{~dB}$ and $3.2 \mathrm{~dB}$, and the relative bandwidth is $11.5 \%$ and $9.7 \%$ respectively. In the second pass band of $5.25 \mathrm{GHz}$, the insertion loss of the simulation results and test results were $1.7 \mathrm{~dB}$ and $2.8 \mathrm{~dB}$, and the relative bandwidth is $6.2 \%$ and $5.1 \%$ respectively. The mesaured results show that the stopband rejection of a bandpass filter is perfect, the insertion loss reach $41 \mathrm{~dB}$ at $3.4 \mathrm{GHz}$ and $4.6 \mathrm{GHz}$ and $6.7 \mathrm{GHz}$, the isolation between two passband is well.

Both filter 1 and filter 2 reveals that the simulated and measured results are in good agreement. Both can meet the requirements in engineering design.

\section{Conclusion}

Two novel compact dual-band filters with low insertion loss are proposed, which have a good performance at $2.4 / 5.2 \mathrm{GHz}$ without any external impedancematching block at the input and output. The designed filters are manufactured and measured, a good agreement between the simulated and measured results are demonstrated.

\section{References}

1. H. Miyake, S. Kitazawa, T. Ishizaki, T. Yamada, and Y. Nagatomi, Aminiaturized monolithic dual band filter using ceramic lamination technique for dual mode portable telephones, in IEEE MTT-S Int. Microw.Symp. Dig., Vol. 2, (1997), pp. 789-792.

2. Fuhong Guan, Xiaowei Sun, Wei Xue, And Jian Zhang. Design of A tunable Dual-Band Filter Using Step-Impedance Resonators with wide Stopband, Global Symposium on Millimeter Waves,( 2008), pp:62-364.

3. M.Makimoto and S.Yamashita, microwave Resonators and Filters for Wireless Communications-Theory and Design. Berlin, (Germany: SpringerVerlag, 2001). 
4. Yue Ping, Zhang and Mei Sun, Dual-Band Microstrip Bandpass Filter Using Stepped-Impedance-Resonators With New Coupling Schemes, IEEE Transactions on microwave theory and technioues.vol.54, pp. 3779-3785.

5. S.Sun and L.zhu. Compact dual-band microstrip bandpass filter without extemal feed, IEEE Microw. Wireless Compon. Lett, (2005), pp.644-646.

6. M.Sagawa, M.Makimoto, S.Yamashita, Geometrical structures and fundamental characteristics of microwave stepped-impedance resonators, IEEE Trans.Microwave Theory Tech., vol.45, (2002), pp.1078-1084.

7. Shun-Yun Lin, Multi-band folded planar monopole antenna for mobile handset,IEEE Trans. Antennas Propagat., vol. 52, (2004),pp. 1790-1794.

8. Z. N. Chen and Y.W. M. Chia, Impedance characteristics of trapezoidal planar monopole antennas, Microw. Opt. Technol. Lett., vol. 27, ( 2000), pp. 120-122.

9. Li Jian kang, Chen Chun Hong, Wu Wen. Design of dual-passband crosscoupled filter using stub-loaded open-loop resonators, Microwave Conference 2009. Asia Pacific, (APMC 2009), pp: 929-932.

10. Lee, C. H., I. C. Wang, and C. I. G. Hsu, Dual-band balanced BPF using $\lambda, / 4$ stepped-impedance resonators and folded feed lines, Journal of Electromagnetic Waves and Applications, Vol. 23, (2009),pp:2441-2449. 